\title{
Evaluating the Nasal Cytology in Acute Rhinosinusitis
}

\author{
Juliana R. Moskowitz ${ }^{\#}$, Jacqueline K. Shaia ${ }^{\# *}$, James R. Schwebach \\ Department of Biology, George Mason University, Fairfax, VA.
}

\begin{abstract}
"Contributed Equally

* Corresponding Author email: jkshaia@icloud.com

Article History

Received: 24 March 2018

Accepted: 2 April 2018

Published: 04 April 2018

Student(s)

- Juliana R. Moskowitz

Academic Year: 2016 / Fall
Course Level: Bachelor
Course Name: B.S. (BioEngineering)
Course year: Final Year

- Jacqueline K. Shaia

Academic Year: 2015 / Spring

Course Level: Bachelor

Course Name: B.S. (Biology)

Course year: Final Year

Mentar(s)

- James R. Schwebach

Rhinitis has been found to have profound direct and indirect negative effects on business including costs attributed to loss of productivity and the amount of over the counter medications consumed. Several other studies have examined the immune system's response in immunocompromised patients by using nasal cytology. This study focused on nasal cytology for a common illness such as acute rhinosinusitis (ARS), in otherwise healthy individuals. Most studies have used nasal cytology to analyze immunocompromised patients, however, this study considers otherwise healthy patients in order to better understand the basic immune response. This study aimed to examine the relationship between neutrophil frequencies found over the course of ARS and symptom severity experienced by subjects. Nasal smears were obtained from subjects at days five, nine, and fourteen of their ARS symptoms. A published symptom survey for ARS was given to participants to provide a qualifying score, signifying a high enough level of symptoms for this study. This survey monitored both emotional and physical symptoms throughout the fourteen-day illness period. Nasal smears were obtained on each visit and stained to observe the different cells present. Wilcoxon Sum Rank Tests were found to be significant between all symptom score visits 1,2 , and 3 $(\mathrm{p}<0.05)$. The average neutrophil counts between all the visits were not significant $(p>0.05)$. Less than ten percent of eosinophils were present in 88.89 percent of subjects. 55.5 percent of subjects did follow an increased amount of neutrophils over the course of their illness. This study reinforces the use of nasal cytology by obtaining nasal smears to demonstrate how neutrophil frequencies change with symptom severity.

Keywords : nasal smear, cell cytology, rhinitis, acute rhinosinusitis, sinusitis, nasal
\end{abstract}

\section{Introduction}

Sinusitis and rhinitis account for hundreds of millions of days of work lost, a decrease in quality of life, and a decrease in productivity. Some studies suggest that granulocytes, such as neutrophils, eosinophils, and basophils, can provide a better understanding of a person's illness [1], [2]. This can be done through

Copyright (C) 2018. The Author(s). Published by AIJR Publisher.

This is an open access article under Creative Commons Attribution-NonCommercial 4.0 International (CC BY-NC 4.0) license, which permits any non-commercial use, distribution, adaptation, and reproduction in any medium, as long as the original work is properly cited. 
observing mucosal samples [2]. Several studies have compared granulocyte frequencies to severe respiratory diseases, such as chronic obstructive pulmonary disease (COPD), asthma, and cystic fibrosis. One such study compared COPD and the amount of neutrophils present with the severity of the disease. They found a more severe form of the disease does not correlate with patients having an increased neutrophil count [3]. Different types of rhinitis have been evaluated through the use of nasal smears obtained through mucosal samples and observed for eosinophil frequencies. "Nonallergic rhinitis with eosinophilia syndrome" or, NARES, has been identified by having more than $20 \%$ eosinophils present through a nasal smear [4]. Other studies are using both neutrophil and eosinophil frequencies to identify different forms of allergic and nonallergic rhinitis [2], [5], [6]. It has been suggested that more than $10 \%$ of inflammatory cells must be eosinophils to indicate allergic rhinitis [2].

There are several forms of rhinitis such as viral and bacterial. Viral rhinosinusitis is known to cause upper respiratory symptoms for up to seven days, whereas acute bacterial rhinosinusitis (ABRS) is more likely when symptoms persist past seven to ten days [7]. Due to the difficulty in differentiating types of rhinitis, a research survey was used to identify subjects specifically with ARS. This survey, "The Sino-Nasal Outcome Test 20," or SNOT 20, is a widely used survey that is a quality of life questionnaire specifically designed for rhinosinusitis patients. This twenty-item survey assists both researchers and physicians in rating severity of the illness and separates symptoms between physical and emotional [8], [9].

The purpose of this study was to quantify the neutrophil frequencies over a fourteen-day period and compare the results with the SNOT 20 survey scores obtained. Mucosal samples were collected to observe the neutrophil and eosinophil frequencies by obtaining nasal smears. Nasal smears were used for several reasons: they are safe, non-invasive, and handled well by patients [2], [5], [7], [10]. They have also been used in other studies to observe the granulocyte frequencies for both allergic rhinitis and nonallergic rhinitis [4], [6], [7], [10].

In this study, we hypothesized that the majority of inflammatory cells present would be neutrophils, as neutrophils have been found to predominate in chronic sinusitis whereas eosinophils tend to have higher presence in allergic rhinitis [2], [10]. We also hypothesized that the higher the original SNOT 20 symptom score, the more neutrophils would be present throughout the illness. The SNOT 20 survey scores should also decrease over time, since rhinosinusitis lasts less than seven to ten days.

\section{Methods}

\subsection{Participants}

The SNOT 20 survey was administered and a specific score was required for individuals to be eligible for this study. Permission to use this survey was obtained from Dr. Piccirillo. Nine subjects were consented and agreed to not take any cold or sinus medications during the fourteen-day experimental period. All subjects were college students between the ages of 19 to 21 years of age and no chronic respiratory illnesses were known at the time of this study. Also, one healthy subject was consented that had five nasal smears conducted on one day. All subjects signed a consent form stating that they understand the minimal risk of their confidentiality and health. Consent was obtained from all subjects and the study was approved by the institutional IRB.

\subsection{Scraping Process}

For each subject, three nasal smears were gathered during the study period on days five, nine, and fourteen. The first smear was completed between days four and six since it takes approximately three to five days for the initial immune response to occur [11]. The second smear occurred between days eight and ten, while the third smear occurred between days thirteen and fifteen. All smears were obtained by utilizing an ASI Rhino-Pro Nasal Mucosal Curette [5], [6]. At each visit, a nasal sample was obtained and the SNOT 20 survey was completed. All scrapings took place in Student Health Services at George Mason University. 
Moskowitz et al., Adv.J. Grad. Res.; Vol. 4, Issue 1, pp: 41-46, July 2018

\subsection{Staining and Covering Process}

Each sample was stained with HANSEL stain. This staining process took place immediately after the smear was obtained.

\subsection{Microscopy and Cell Counting}

All samples were observed under a light microscope at 40X, and then pictures were taken at 100X oil immersion. Ten fields were acquired per nostril and were hand-counted for neutrophils, eosinophils, and degranulation. One field contains $50 \%$ or more of a slide covered in cells, $25 \%$ coverage counted as half a field, while no field was counted for less than $25 \%$ [10]. Figure 1 represents a completely covered field. Both co-investigators counted the different cells and the values were averaged. To count a neutrophil, at least two of the lobes in the cell must be clearly present in the field. Graphs were made to analyze the overall sum of neutrophils for each visit, along with symptom scores. Wilcoxon Sum Rank tests were performed on the cell

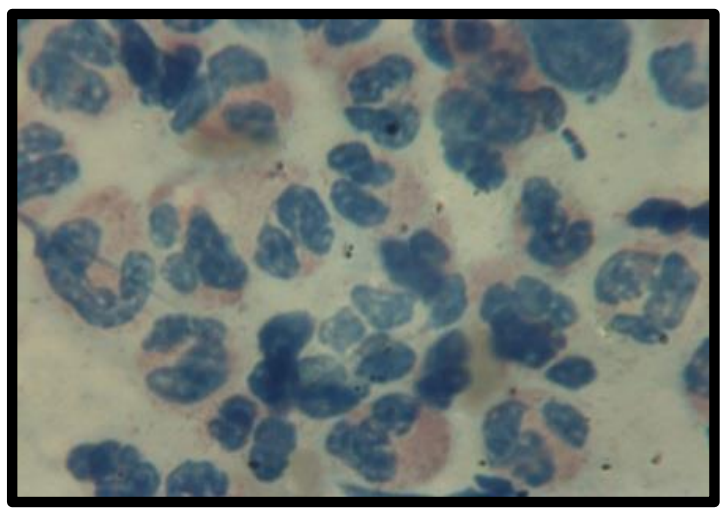

Figure 1: A visit three image of neutrophils frequencies and symptom scores data. This test was used due to the small sample size along with the only assumption being randomized data. A p-value of $\mathrm{p}<0.05$ was considered significant.

\section{Results}

One hundred percent of the subjects had a decrease in symptom scores over the fourteen-day period (Figure 2). Wilcoxon Sum Rank Tests were performed and found to be significant between visit one and visit three for overall symptom scores, or the total score calculated from the SNOT twenty survey ( $p$ value $=0.0003$ ) (Table 1). The comparisons between visit one and two and visit two and three were also found to be significant $(p$ values $=0.001$ and 0.03$)$.

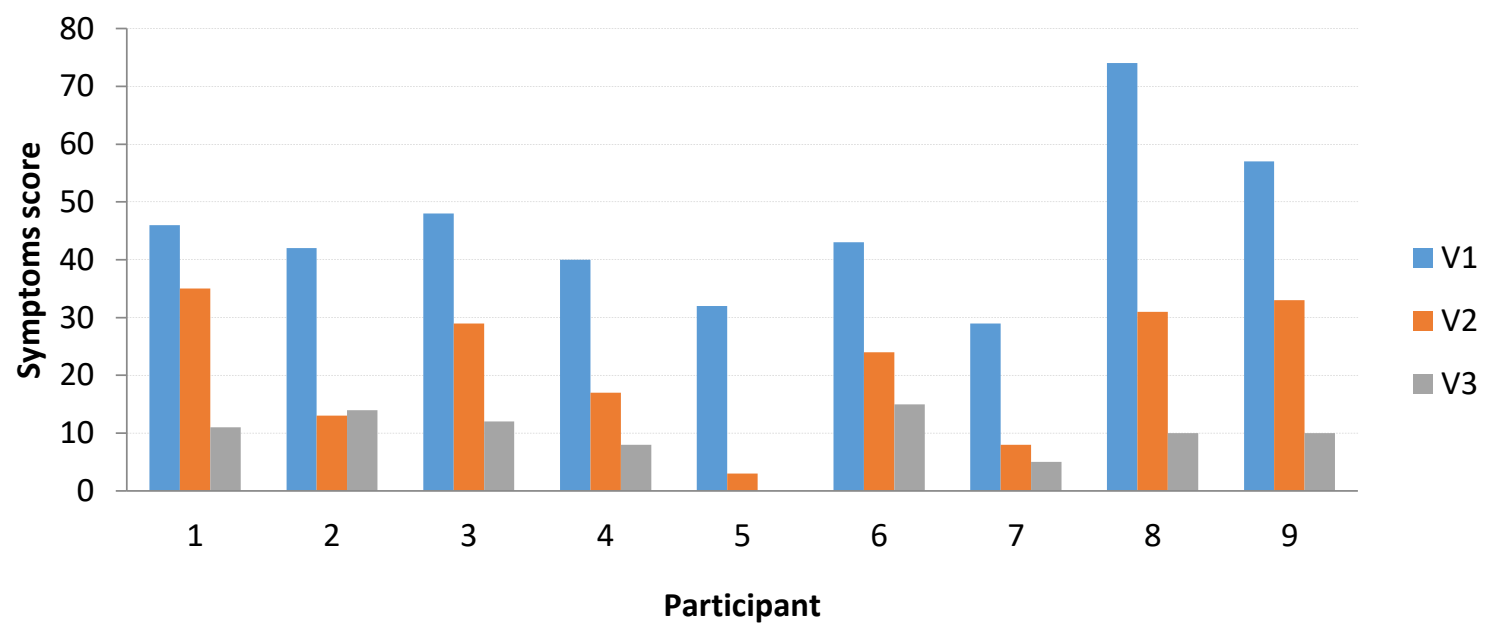

Figure 2: Symptom score per patient per visit showing only the first ten symptoms that are the physical not emotional.

Table 1: Wilcoxon Sum Rank Tests of Symptoms scores and comparisons between physical and emotional symptoms.

\begin{tabular}{|l|r|r|r|r|}
\hline $\begin{array}{l}\text { Wilcoxon Sum Rank Tests } \\
\text { (p values) }\end{array}$ & $\begin{array}{l}\text { Total Symptom } \\
\text { Scores }\end{array}$ & $\begin{array}{l}\text { Average Physical } \\
\text { Symptoms }\end{array}$ & $\begin{array}{l}\text { Average Emotional } \\
\text { Symptoms }\end{array}$ & \multicolumn{1}{l|}{$\begin{array}{l}\text { Neutrophil } \\
\text { Counts }\end{array}$} \\
\hline V1 vs. V3 & 0.0003461 & 0.0004542 & 0.0001517 & 0.356 \\
\hline V1 vs. V2 & 0.001987 & 0.0138 & 0.000986 & 0.691 \\
\hline V2 vs. V3 & 0.03388 & 0.04817 & 0.002614 & 0.2886 \\
\hline
\end{tabular}


Evaluating the Nasal Cytology in Acute Rbinosinusitis

Symptoms were broken down into two groups: physical and emotional [9], [12]. The physical symptoms were found to be significant between visit one and three $(p$ value $=0.0004)$. Significance was also found between visit one and two, and visit two and three ( $p$ values $=0.01 ; 0.04$, respectively). For the emotional symptoms, significance was found between visit one and three and visit one and two $(p$ values .0001; 0.001, respectively). Average neutrophil counts were not significant between any of the visits ( $p$ value $>0.05)$. As can be seen in Figure 4, the range of neutrophil counts per symptom score varied greatly between subjects.

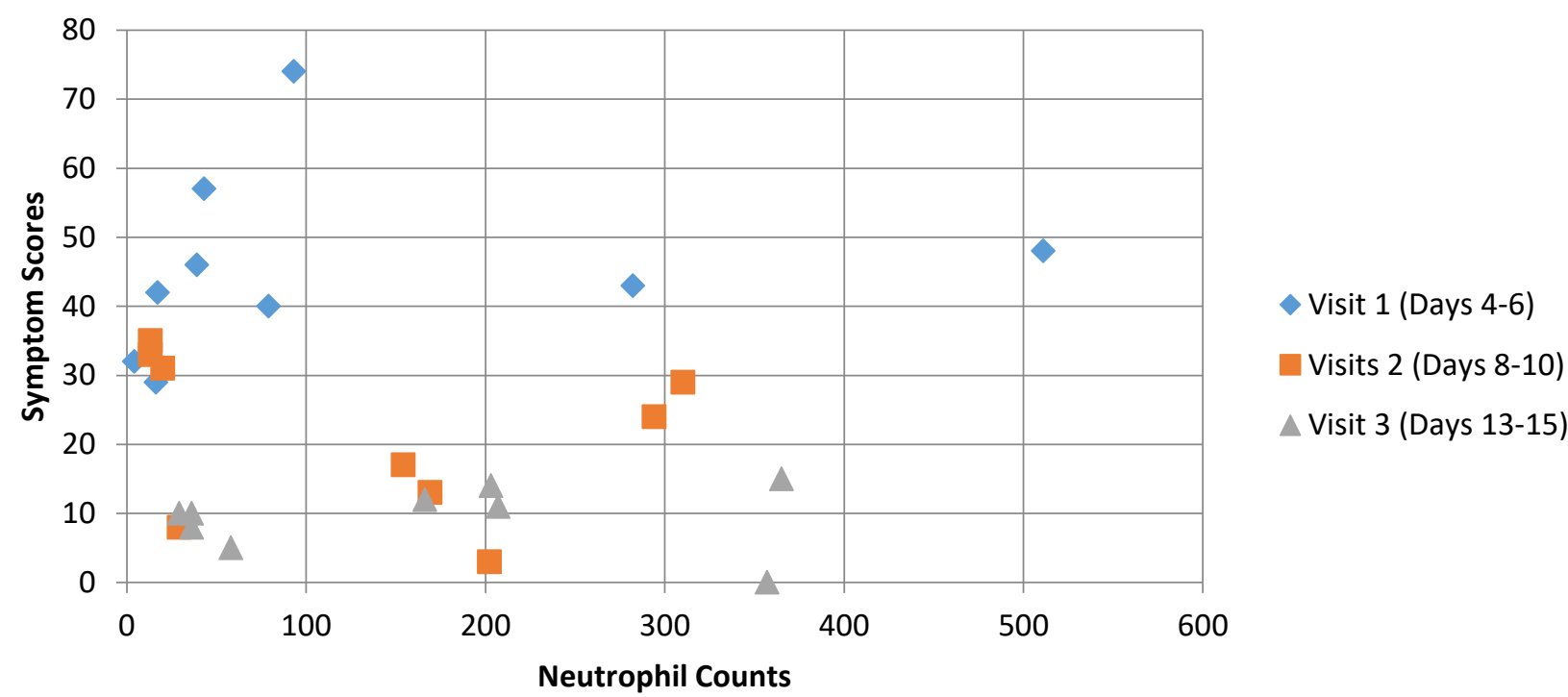

Figure 3: Symptom scores versus neutrophil counts for every patient on visit 1, visit 2, and visit 3 .

Figures 4 and 5 represent the percentage of neutrophils and eosinophils found in each nasal smear per subject. This was based on the overall inflammatory cells counted. The majority of subjects were seen to have an increase in neutrophils throughout their illness (Figure 4). In almost all subjects, eosinophils were not seen. However, one subject was found to have greater than twenty percent of eosinophils present on multiple nasal smears (Figure 5).

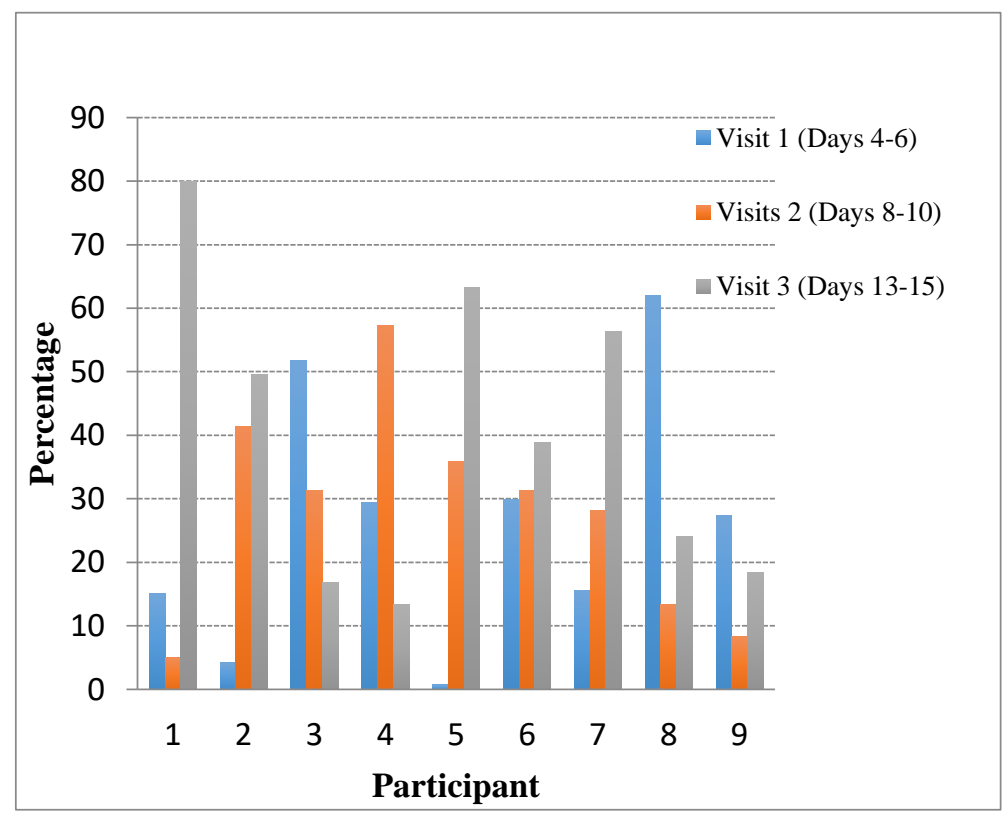

Figure 4: Percentage of neutrophils found in each smear per each participant.

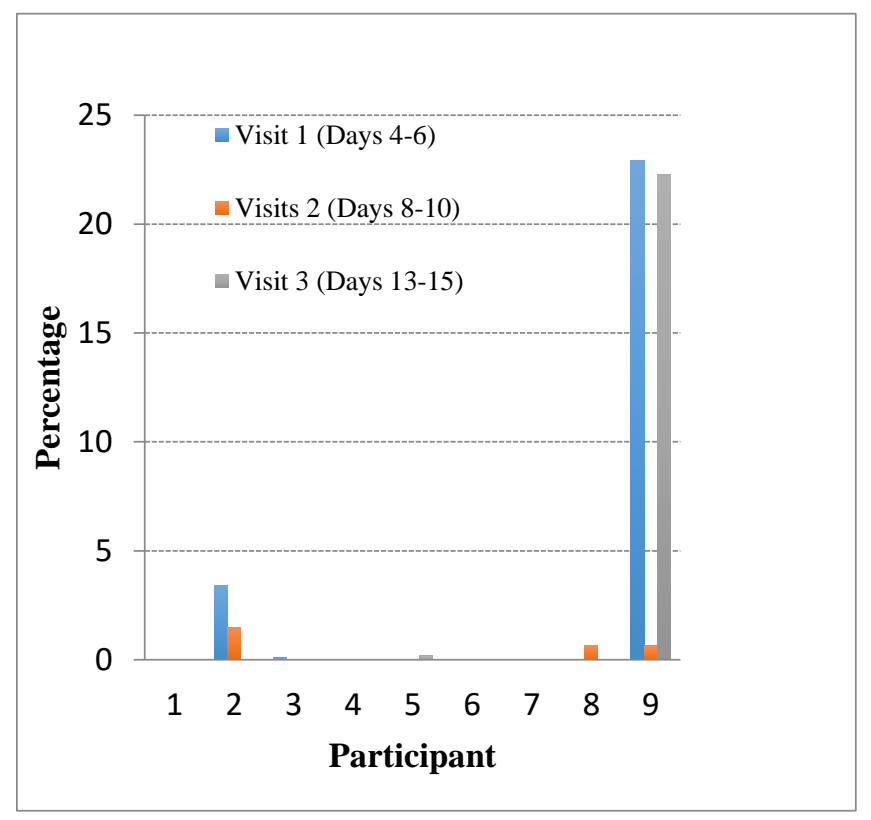

Figure 5: Percentage of neutrophils found in each smear per each participant. 


\section{Discussion}

In this study we were able to observe many different aspects of ARS. All symptoms scores were evaluated and found to be significant for both the emotional and physical symptoms and the overall SNOT 20 score. This information provides a glimpse into the immune system and shows improvements in symptoms and overall wellness in patients with ARS [8]. Overall, 55.5 percent of the subjects did follow an increase in neutrophil frequencies throughout their illness. Minimal subjects had the highest neutrophil frequencies on their first or second visit, meaning they may have had a stronger immune response earlier in the illness (Figures: 3,4). A greater percentage of neutrophils present later in a subject's illness may have been found because their immune system was more active. This would cause subjects symptom scores to decrease as their symptoms were improving. With a larger sample size, a potential trend may become significant.

In this investigation it was important to validate that each patient did have ARS not only for consistency but also to make sure we could create a study that specifically looked at this type of rhinitis. This baseline study provides a greater look into a basic immune response specifically for ARS. Several studies have worked to focus on diagnosing the specifics of rhinitis. NARES, has been identified by having more than $20 \%$ eosinophils present through a nasal smear [2], [4]. It has been suggested that more than $10 \%$ of inflammatory cells must be eosinophils to indicate allergic rhinitis [2]. In this study there were less than ten percent of eosinophils in 88.89 percent of subjects. This indicates that ARS was likely the cause of the sickness as both NARES and allergic rhinitis have more than $10 \%$ of eosinophils to specify their illness. One subject did present with increased eosinophils over $20 \%$ which signifies that they may have had allergic rhinitis.

There are few studies that have investigated neutrophil counts as a result of having a common cold. Most research has been used for allergic rhinitis and has shown that nasal smears provide an accurate look into cell cytology [10]. Both bacterial and viral rhinosinusitis studies that have included neutrophil counts are limited and importantly, the majority of cell cytology studies used immunocompromised patients. Therefore, this research shows baseline neutrophil counts in otherwise healthy human rhinosinusitis subjects and validates the use of nasal cytology for rhinitis.

\section{Conclusion}

In conclusion, this study reinforces the validity of cell cytology through the use of nasal smears by demonstrating how neutrophil frequencies change with symptom severity, in the absence of any treatment. Overall, a majority of subjects had an increase in neutrophil frequencies throughout their illness. Additionally, all of the patients from their first visit to third visit had a significant decrease in not only their total symptoms, but also physical and emotional symptoms. An increased routine use of nasal smear cytology in rhinitis patients may diminish the inappropriate use of antibiotics in this patient population for several reasons including correct diagnosis of allergies, bacterial rhinitis, and viral rhinitis.

\section{Declaration}

\subsection{Acknowledgements}

The authors wish to acknowledge Dr. Bruce K. Rubin, M.Engr., M.D., M.B.A., FRCPC for his mentoring and guidance in the preparation and performance of this study. We would also like to acknowledge Dr. Joseph J. Pancrazio and the bioengineering department at George Mason University for the use of their lab and assistance throughout the project.

\subsection{Study Limitations}

One limitation to this study is the small sample size. There was a difficulty in finding subjects who would consent to taking no medications while being sick. Another difficulty was the narrow age range. With this research being done at a university, there was not a large age range to allow for a comprehensive 
investigation into the immune system's nasal cellular response at all ages. Larger sample sizes and a broader age range would benefit further studies.

\subsection{Ethical Approval}

This research was approved by George Mason University’s Institutional Review Board, 630561-1.

\subsection{Informed Consent}

Informed consent was completed for all participants.

\subsection{Funding Source}

Funding was received from the Office of Student Scholarship, Creative Activities, and Research at George Mason University through their Undergraduate Research Scholars Program.

\subsection{Competing Interests}

There are no competing interests for any of the authors and co-authors on this paper.

\section{How to Cite this Article:}

J. Moskowitz, J. Shaia, and J. Schwebach, "Evaluating the Nasal Cytology in Acute Rhinosinusitis", Advanced Journal of Graduate Research, vol. 4, no. 1, pp. 41-46, Apr. 2018. doi: 10.21467/ajgr.4.1.41-46

\section{References}

[1] B. Geering, C. Stoeckle, S. Conus, and H.-U. Simon, "Living and dying for inflammation: neutrophils, eosinophils, basophils.," Trends Immunol., vol. 34, no. 8, pp. 398-409, Aug. 2013.

[2] H. S. Lee, Y. Majima, Y. Sakakura, J. Shinogi, S. Kawaguchi, and B. W. Kim, "Quantitative Cytology of Nasal Secretions Under Various Conditions," Laryngoscope, vol. 103, no. 5, p. 533???537, May 1993.

[3] T. Yoshikawa et al., "Impaired Neutrophil Chemotaxis in Chronic Obstructive Pulmonary Disease," Am. J. Respir. Crit. Care Med., vol. 175, no. 5, pp. 473-479, Mar. 2007.

[4] C. Bachert, "Persistent rhinitis - allergic or nonallergic?," Allergy, vol. 59, no. s76, pp. 11-15, Jan. 2004.

[5] M. Gelardi, M. L. Fiorella, G. Leo, and C. Incorvaia, "Cytology in the diagnosis of rhinosinusitis," Pediatr. Allergy Immunol., vol. 18, no. s18, pp. 50-52, Nov. 2007.

[6] G. Matteo et al., "Nasal cytology in children: recent advances," Ital. J. Pediatr., vol. 38, no. 1, p. 51, Sep. 2012.

[7] M. S. Dykewicz and D. L. Hamilos, "Rhinitis and sinusitis.," J. Allergy Clin. Immunol., vol. 125, no. 2 Suppl 2, pp. S103-15, Feb. 2010 .

[8] D. A. E. Dietz de Loos, C. L. Segboer, A. Gevorgyan, and W. J. Fokkens, "Disease-Specific Quality-of-Life Questionnaires in Rhinitis and Rhinosinusitis: Review and Evaluation,” Curr. Allergy Asthma Rep., vol. 13, no. 2, pp. 162-170, Apr. 2013.

[9] J. F. Piccirillo, M. G. Merritt, and M. L. Richards, "Psychometric and Clinimetric Validity of the 20-Item Sino-Nasal Outcome Test (Snot-20)," Otolaryngol. Neck Surg., vol. 126, no. 1, pp. 41-47, Jan. 2002.

[10] A. Özgür, S. Arslanoğlu, D. Etıt, U. Demıray, and H. K. Önal, "Comparison of nasal cytology and symptom scores in patients with seasonal allergic rhinitis, before and after treatment," J. Laryngol. Otol., vol. 125, no. 10, pp. 1028-1032, Oct. 2011.

[11] K. Murphy, Janeway's immunobiology. 2017.

[12] M. A. Pynnonen, H. M. Kim, and J. E. Terrell, "Validation of the Sino-Nasal Outcome Test 20 (SNOT-20) domains in nonsurgical patients," Am. J. Rhinol. Allergy, vol. 23, no. 1, pp. 40-45, Jan. 2009.

Publish your research article in AIJR journals-

$\checkmark \quad$ Online Submission and Tracking

$\checkmark \quad$ Peer-Reviewed

$\checkmark$ Rapid decision

$\checkmark \quad$ Immediate Publication after acceptance

$\checkmark \quad$ Articles freely available online

$\checkmark \quad$ Retain full copyright of your article.

Submit your article at journals.aijr.in
Publish your books with AIJR publisher-

$\checkmark \quad$ Publish with ISBN and DOI.

$\checkmark$ Publish Thesis/Dissertation as Monograph.

$\checkmark \quad$ Publish Book Monograph.

$\checkmark \quad$ Publish Edited Volume/ Book.

$\checkmark$ Publish Conference Proceedings

$\checkmark \quad$ Retain full copyright of your books.

Submit your manuscript at books.aijr.org 